Mini Review

\title{
Interventions and assessments of bilateral upper extremity movement for stroke patients
}

\section{Introduction}

Recovery of bilateral upper extremity function is a core element for stroke patients with hemiplegia. Because most activities of daily living are included bilateral upper extremity tasks, stroke patients with affected upper limb may have difficulty performing activities of daily living tasks. ${ }^{1}$ This result can negatively effect on participation of daily living and quality of life. Thus, most occupational therapists provide bilateral upper extremity training with stroke patients in rehabilitation center or hospitals. ${ }^{2}$

There are three main types of bilateral upper extremity training; functional task training (FTT), bilateral training with rhythmic auditory cues (BATRAC), and robot-assisted training (RAT). ${ }^{3}$ These bilateral upper extremity therapies can activate with affected hemisphere, especially the secondary motor areas, by interhemispheric connections. ${ }^{4}$ The positive change of neural mechanism leads to improvement of complementary bilateral functional skills, as well as, functional level of paretic arm. ${ }^{5}$

We will review the current three bilateral training studies including effect and standard tool for assessing post bilateral training.

\section{Interventions}

\section{Functional task training (FTT)}

FTT is consisted of bilateral tasks based on activities of daily living such as making coffee, typing, cutting fruit et al. In a comparative study (FTT versus occupational therapy) including 30 participants with hemiplegic stroke, it was investigated on the effectiveness of bilateral arm training for improving upper limb function using FuglMeyer assessment (FMA) and Box and Block Test (BBT) assessment. The result of this study was reported that FTT using bilateral upper extremity with general occupational therapy might be more effective than occupational therapy alone for improving upper extremity function. ${ }^{6}$

\section{Bilateral training with rhythmic auditory cues (BATRAC)}

BATRAC is a protocol providing bilateral arm symmetrical and asymmetrical movement accompanied by rhythmic auditory cueing. Shahine and Shafshak (2014) investigated about effects of BATRAC on both upper extremity motor performance and motor evoked potential comparing traditional therapeutic exercise for patients with stroke. Outcome measures were included FMA and Transcranial magnetic stimulation. The result was found that there was a significant increase in motor performance and motor evoked potential parameters in the BATRAC group. ${ }^{7}$

\section{Robot-assisted training (RAT)}

RAT is an intervention approach that assists high-intensity, highrepetition, and task-specific training. In study used the Bi-Manu-

\author{
Volume 7 Issue 5 - 2017 \\ Lee Joo-Hyun,' Jeon Yu-Jin ${ }^{2}$ \\ 'Chosun University, Korea \\ ${ }^{2}$ National Rehabilitation Center, Korea \\ Correspondence: Lee Joo-Hyun, Chosun University, 309, \\ Pilmun-daero, Dong-gu, Gwangju, Republic of Korea; \\ Email hy100za@hanmail.net \\ Received: March 15, 2017 | Published: March 30, 2017
}

Track as robot tool, participants received RAT that provides bilateral training of wrist and forearm movements. The primary outcome was kinematic variables, and secondary outcomes were included Wolf Motor Function Test (WMFT) for motor function assessment. The result showed that it was significantly improved upper extremity motor control. ${ }^{8}$

\section{Discussion}

The review indicated that bilateral upper extremity training can dramatically improve motor function of stroke patients with hemiplegia. But most studies were used instruments for assessing motor function of hemiplegic side, not bilateral upper extremity function. Assessment tools used for unilateral (paretic) upper extremity have low sensitivity to changes in functional movement level of bilateral upper extremity. ${ }^{9}$ It is very important to use instrument for evaluating movement pattern of bilateral upper extremity exactly. Further research is needed to evaluate the accuracy of outcome bilateral upper extremity interventions.

\section{Acknowledgments}

None.

\section{Conflicts of interest}

None.

\section{References}

1. Lum PS, Mulroy S, Amdur RL, et al. Gains in upper extremity function after stroke via recovery or compensation: potential differential effects on amount of real-world limb use. Top Stroke Rehabil. 2009;16(4):237-253.

2. Stoykov ME, Corcos DM. A review of bilateral training for upper extremity hemiparesis. Occup Ther Int. 2009;16(3-4):190-203. 
3. Wolf A, Scheiderer R, Napolitan N, et al. Efficacy and task structure of bimanual training post stroke: systematic review. Top Stroke Rehabil. 2009;21(3):181-196.

4. Hijmans JM, Hale LA, Satherley JA, et al. Bilateral upper-limb rehabilitation after stroke using a movement-based game controller. $J$ Rehabil Med. 2011;48(8):1005-1014.

5. Waller SM, Whitall J. Bilateral arm training: why and who benefits? Neuro Rehabilitation. 2008;23(1):29-41.

6. Lee MJ, Lee JH, Koo HM, et al. Effectiveness of bilateral arm training for improving extremity function and activities of daily living performance in hemiplegic patients. J Stroke Cerebrovasc Dis pii: 2017;S1052-3057(16)30605-X.
7. Shahine EM, Shafshak TS. The effect of repetitive bilateral arm training with rhythmic auditory cueing on motor performance and central motor changes in patients with chronic stroke. Egyptian Rheumatology and Rehabilitation. 2014;41(1):8-13.

8. Hsieh YW, Liing RJ, Lin KC, et al. Sequencing bilateral robot-assisted arm therapy and constraint-induced therapy improves reach to press and trunk kinematics in patients with stroke. J Neuroeng Rehabil. 2016;13(1):31.

9. Wolf A, Scheiderer R, Napolitan, N, et al. Efficacy and task structure of bimanual training post stroke: a systematic review. Top Stroke Rehabil. 2014;21(3):181-196. 